\title{
Flexible Bronchoscopy Is Safe and Effective in Adult Subjects Supported With Extracorporeal Membrane Oxygenation
}

\author{
Nirmal S Sharma MD, Timothy Peters MD, Tejaswini Kulkarni MD MPH, Charles W Hoopes MD, \\ Scott C Bellot MD, Keith M Wille MD MSPH, and Enrique Diaz-Guzman MD
}

\begin{abstract}
BACKGROUND: Previous studies have demonstrated the safety of flexible bronchoscopy (FB) in mechanically ventilated subjects. However, the safety of $\mathrm{FB}$ in adult subjects receiving extracorporeal membrane oxygenation (ECMO) has not been described previously. METHODS: A retrospective review was conducted of all adult subjects who underwent $\mathrm{FB}$ while receiving ECMO support at the University of Alabama at Birmingham Hospital from January 1, 2013, to December 31, 2014. Physiologic variables, pre- and post-FB ECMO, and ventilator settings were recorded. RESULTS: 79 adult subjects underwent FB receiving ECMO with a total of 223 bronchoscopies. The most common indications for bronchoscopy included diagnostic evaluation of infection in subjects with pneumonia (29\%) and clearance of excessive secretions $(22 \%)$. In $70 \%$ of subjects, moderate or greater amounts of secretions were noted. FB yielded positive culture data in 37 subjects $(47 \%)$, which resulted in a change to the antibiotic regimen in 14 subjects $(38 \%)$ with positive culture data. No significant differences in mean $\mathrm{P}_{\mathrm{aO}_{2}} / \mathrm{F}_{\mathrm{IO}_{2}}$, mean ECMO flow, mean sweep gas, ventilator settings, or hemodynamic parameters (heart rate, oxygen saturation, and mean blood pressure) were noted before and after FB. Complications were mild and transient: blood-tinged secretions after FB in $21 \%$ cases, which resolved spontaneously, intraprocedural hypoxemia in $2.2 \%$ of cases, and dysrhythmia in $<1 \%$ of cases. There were no episodes of ECMO cannula dislodgement or inadvertent extubation. CONCLUSIONS: FB can be used safely in adult subjects supported with ECMO and is not associated with significant hemodynamics changes, bleeding, or mechanical complications during ECMO support. Key words: ECMO; bronchoscopy; ARDS; cardiorespiratory failure; veno-venous ECMO; veno-arterial ECMO. [Respir Care 2016;61(5):646-651. (C) 2016 Daedalus Enterprises]
\end{abstract}

\section{Introduction}

In the past decade, there has been an increase in the utilization of extracorporeal membrane oxygenation (ECMO) to support critically ill patients with cardiopul-

\footnotetext{
Drs Sharma, Peters, Kulkarni, Wille, and Diaz-Guzman are affiliated with the Division of Pulmonary and Critical Care Medicine, and Drs Hoopes and Bellot are affiliated with the Division of Cardiothoracic Surgery, University of Alabama at Birmingham, Birmingham, Alabama.

The authors have disclosed no conflicts of interest.

Correspondence: Enrique Diaz-Guzman MD, Division of Pulmonary and Critical Care Medicine, University of Alabama at Birmingham, Birmingham, AL 35294-0006. E-mail: diaze@uab.edu.
}

DOI: $10.4187 /$ respcare.04456 monary failure. ${ }^{1,2}$ ECMO-supported patients often have respiratory infections and increased airway secretions complicating the course of their illness. ${ }^{3}$ To aid in the diagnosis of respiratory infections and facilitate secretion clearance, flexible bronchoscopy (FB) has become a necessary tool in modern critical care practice. ${ }^{4,5}$ Although FB is a relatively safe procedure in most patients, it can be associated with complications, such as worsening hypoxemia, endotracheal tube (ETT) dislodgement, and airway trauma, in critically ill subjects receiving mechanical ventilation. ${ }^{6-12}$ Additionally, FB may entail a greater risk of complications in ECMO patients due to the use of systemic anticoagulation and greater degree of illness in these patients. ${ }^{13,14}$ Previous studies have demonstrated the safety of FB in subjects receiving mechanical ventilation. ${ }^{15,16}$ However, its safety profile in adult patients supported with ECMO is not well established. 
In this retrospective study, we present our experience with FB in adult subjects supported with ECMO and analyze its safety and utility in this cohort. We hypothesize that FB is a safe and well-tolerated procedure for patients receiving ECMO support.

\section{Methods}

\section{Subject Population and Data Collection}

We performed a retrospective review of all ECMO subjects who underwent FB for any indication at the University of Alabama Hospital at Birmingham from January 1, 2013, to December 31, 2014. This project was reviewed and approved by the University of Alabama Hospital at Birmingham institutional review board for human research (Protocol X140909006).

\section{Subject Selection}

A total of 141 subjects were supported with ECMO for any indication during the period of interest. Of these, 79 subjects underwent a total of 223 FBs.

\section{Outcome Measures}

Variables collected before and after bronchoscopy included demographics, FB indication, vital signs, ventilator settings, ECMO settings, chest radiograph changes, and complications. Vital signs and ventilator and ECMO settings recorded immediately before and $2 \mathrm{~h}$ after the procedure were utilized for this study. Chest radiograph reports as interpreted by attending radiologists before ECMO and the first after FB were used for analysis.

\section{ECMO Management}

At our institution, an internal jugular double-lumen cannula is used for venovenous (VV) support, and a jugulofemoral route is preferred for venoarterial (VA) ECMO in a majority of patients. Less commonly, a mixed configuration (combination of the above) is utilized, according to the patient's condition and oxygen requirement. Anticoagulation is managed per protocol ${ }^{17}$ and monitored using anti-factor $\mathrm{Xa}$ and thromoboelastography. An anti-factor $\mathrm{Xa}$ of $0.2-0.4 \mathrm{IU} / \mathrm{ml}$ and $\mathrm{R}$ time on thromoboelastography of 2.5-3 times the control value are usually maintained. No changes were made to anticoagulation before or after FB. Either a supervised critical care trainee or the attending physician performed FB. In addition, a critical care nurse, respiratory therapist, and perfusionist were also present during the procedure.

\section{QUICK LOOK}

\section{Current knowledge}

In the past decade, there has been an increased utilization of ECMO to support patients with severe cardiorespiratory failure. Flexible bronchoscopy has become an important tool to diagnose respiratory infections and manage airway secretions in these critically ill patients receiving ECMO. Although the safety of flexible bronchoscopy in mechanically ventilated patients has been well described, its safety and utility have not been established in adult patients supported with ECMO.

\section{What this paper contributes to our knowledge}

Flexible bronchoscopy is safe in adult subjects supported with ECMO. Flexible bronchoscopy is not associated with significant hemodynamics changes, bleeding, and/or mechanical complications during ECMO support. Flexible bronchoscopy during ECMO support facilitates pulmonary secretion clearance and improves antibiotic selection in subjects with respiratory infections.

\section{Flexible Bronchoscopy}

At our institution, FB is performed via the ETT, tracheostomy, or the oral route (in extubated patients) while the patient is receiving ECMO support. The ventilator $\mathrm{F}_{\mathrm{IO}_{2}}$ is transiently increased to $100 \%$ for the duration of the procedure and lowered back to baseline as tolerated after the procedure. Bronchoalveolar lavage (BAL) is performed if indicated. Both therapeutic (distal tip $5.9 \mathrm{~mm}$, insertion tube diameter of $6.0 \mathrm{~mm}$, working channel of $3.0 \mathrm{~mm}$ ) and diagnostic bronchoscopes (distal tip $4.8 \mathrm{~mm}$, insertion tube diameter of $4.9 \mathrm{~mm}$, working channel of $2.0 \mathrm{~mm}$ ) are used for FB in ECMO patients based on the clinical indications. In patients receiving mechanical ventilation, usually an ETT and tracheostomy tube size $\geq 8 \mathrm{~mm}$ in diameter is preferred for performing FB. Before FB, patients are premedicated with sedative agents, such as fentanyl, midazolam, or propofol, as clinically determined by the physician. Occasionally, in patients with recent lung transplants who are receiving ECMO, sedation-free awake bronchoscopy is performed. Lidocaine is atomized or nebulized via the ETT/tracheostomy before scope insertion. Most FBs are performed in the recumbent or semirecumbent position. Mean duration of FB is usually 5-15 min, depending on the indication of FB, need for BAL, and amount of secretions present. Although the average time period for each bronchoscope insertion is $60-90 \mathrm{~s}$, this may be increased to $2-5 \mathrm{~min}$ if $\mathrm{BAL}$ is being performed. Culture specimens 


\section{Flexible Bronchoscopy Is Safe With ECMO}

are obtained from bronchial washes, BAL $(100 \mathrm{~mL}$ of sterile saline is used in 4 sequential aliquots of $25 \mathrm{~mL}$ each) or sometimes using a protected specimen brush. Although most of our patients are supported with the pressure control continuous mandatory ventilation mode during ECMO support, we have not found it to be superior (in terms of reduced complication rates) compared with other modes of ventilation during $\mathrm{FB}$.

\section{Statistical Analysis}

We performed descriptive analyses of measured variables using proportions, means $\pm \mathrm{SD}$, or medians with interquartile ranges as appropriate. Changes in parameters before and after FB were compared using an unpaired 2 -way $t$ test or Mann-Whitney test. A $P$ value of $<.05$ was considered significant. Statistical analyses were performed with JMP 10.0 (SAS Institute, Cary, North Carolina).

\section{Results}

\section{Baseline Characteristics}

Of 141 patients supported with ECMO during the study period, 79 underwent $\mathrm{FB}$ and were included in the analysis. A total of $223 \mathrm{FB}$ procedures were performed: 76 (34.1\%) of subjects underwent one FB, 48 (21.4\%) had 2 FBs, 34 (15.1\%) had $3 \mathrm{FBs}$, and $65(29.4 \%)$ had $\geq 4$ procedures. Indications for ECMO support and use of FB are detailed in Table 1. Clearance of excessive secretions and identification of pathogens involved in pneumonia were the most common indications for FB. Median age was 46 y (range 19-83 y). A majority of subjects (59\%) were male. Most subjects (71\%) were supported using VV ECMO, whereas $23 \%$ were supported by VA ECMO and $6 \%$ by a mixed ECMO configuration. A low tidal volume ventilation strategy was preferred for all subjects. Pressure control continuous mandatory ventilation was the ventilatory mode used in $70 \%$ of subjects (Table 2). Whereas a majority of bronchoscopies $(56 \%)$ were performed via the ETT, $42 \%$ were performed via a tracheostomy, and $2 \%$ were performed via the oral route. BAL was performed in $34 \%$ of the procedures. Mean $\mathrm{P}_{\mathrm{aO}} / \mathrm{F}_{\mathrm{IO}_{2}}$ ratio before ECMO initiation was $63 \pm 22.1$. Qualitative bronchoscopy findings included no secretions in $4 \%$, mild secretions in $25 \%$, and moderate or greater secretions in $71 \%$.

\section{Bronchoscopy Outcomes}

Mean pre-FB $\mathrm{P}_{2 \mathrm{O}_{2}} / \mathrm{F}_{\mathrm{IO}_{2}}$ for all subjects was $164 \pm 133$. The pre-FB $\mathrm{P}_{\mathrm{aO}} / \mathrm{F}_{\mathrm{IO}}$ was significantly lower in subjects supported with VV ECMO (149 \pm 109$)$ as compared with those supported with VA ECMO $(200 \pm 156)$ and mixed ECMO configurations $(229 \pm 224), P=.009$. Overall,
Table 1. Indications for Initiation of Extracorporeal Membrane Oxygenation Support and Use of Flexible Bronchoscopy

\begin{tabular}{lc}
\hline \multicolumn{1}{c}{ Indications } & Percentage \\
\hline For ECMO & \\
Bacterial pneumonia & 29 \\
Influenza pneumonia & 18 \\
Cardiogenic shock & 13 \\
Combined bacterial and viral pneumonia & 8 \\
Respiratory failure not otherwise specified & 6 \\
Trauma & 6 \\
Bridge to transplantation & 6 \\
Postoperative complication & 6 \\
Other & 3 \\
Pulmonary embolus & 5 \\
For FB & \\
Pneumonia & 29 \\
Abnormal chest radiograph & 20 \\
Excessive secretions & 22 \\
Atelectasis & 7 \\
Tracheostomy placement & 6 \\
Hemoptysis & 5 \\
Refractory hypoxemia & 4 \\
Diffuse parenchymal disease & 4 \\
Airway inspection & 4 \\
Dislodged endotracheal tube & 2 \\
\hline ECMO = extracorporeal membrane oxygenation & 1 \\
FB flexible bronchoscopy & \\
\hline
\end{tabular}

Table 2. Mode of Ventilation Used During Extracorporeal Membrane Oxygenation Support

\begin{tabular}{lc}
\hline Mode of Ventilation & Percentage of S \\
\hline PC-CMV & 70 \\
VC-CMV & 22 \\
PSV & 3 \\
PC-IMV & 3 \\
VC-IMV & 1 \\
& \\
\hline 1\% of subjects were extubated and off mechanical ventilation while on extracorporeal \\
membrane oxygenation support. \\
PC-CMV = pressure control continuous mandatory ventilation \\
VC-CMV = volume control continuous mandatory ventilation \\
PC-IMV = pressure control intermittent mandatory ventilation \\
VC-IMV = volume control intermittent mandatory ventilation \\
PSV = pressure support ventilation
\end{tabular}

mean $\mathrm{P}_{\mathrm{aO}_{2}} / \mathrm{F}_{\mathrm{IO}_{2}}$ did not change significantly after $\mathrm{FB}$ $(178 \pm 163, P=.35)($ Table 3$)$.

Mean pre-FB ECMO flow was similar for all ECMO configurations. Overall, no significant change in ECMO flow was noted before versus after FB. However, post-FB ECMO flow was significantly higher in subjects with the mixed configuration $(4.3 \pm 0.83 \mathrm{~L} / \mathrm{min})$ group as compared with the VA $(3.74 \pm 0.96 \mathrm{~L} / \mathrm{min})$ and $\mathrm{VV}(3.8 \pm 0.6$ $\mathrm{L} / \mathrm{min})$ ECMO groups $(P=.009)$. Likewise, baseline 


\section{Flexible Bronchoscopy Is Safe With ECMO}

Table 3. Bronchoscopy Outcomes on Extracorporeal Membrane Oxygenation Support

\begin{tabular}{lccc}
\hline \hline & Pre-FB & 2 h Post-FB & $P$ \\
\hline Mean $\mathrm{P}_{\mathrm{aO}_{2}} / \mathrm{F}_{\mathrm{IO}_{2}}$ & & & \\
Overall & $164 \pm 133$ & $178 \pm 163$ & .35 \\
VV subjects & $149 \pm 109$ & $156 \pm 118$ & .54 \\
VA subjects & $200 \pm 156$ & $203 \pm 168$ & .93 \\
$\quad$ Mixed configuration subjects & $229 \pm 224$ & $317 \pm 343$ & .36 \\
Mean ECMO flow, L/min & & & \\
Overall & $3.85 \pm 0.69$ & $3.86 \pm 0.70$ & .97 \\
VV subjects & $3.84 \pm 0.60$ & $3.83 \pm 0.60$ & .93 \\
VA subjects & $3.86 \pm 0.90$ & $3.74 \pm 0.96$ & .58 \\
Mixed configuration subjects & $3.99 \pm 0.89$ & $4.3 \pm 0.83$ & .24 \\
Mean ECMO sweep gas flow, L/min & & & \\
Overall & $4.01 \pm 2$ & $4.0 \pm 2.05$ & .97 \\
VV subjects & $4.13 \pm 1.97$ & $4.11 \pm 2.01$ & .92 \\
VA subjects & $2.84 \pm 1.62$ & $2.87 \pm 1.59$ & .94 \\
Mixed configuration subjects & $5.4 \pm 1.99$ & $5.4 \pm 2.2$ & .94 \\
Mean ventilator $\mathrm{F}_{\mathrm{IO}}$ & $0.68 \pm 0.23$ & $0.64 \pm 0.22$ & .09 \\
Mean PEEP, cm $\mathrm{H}_{2} \mathrm{O}$ & $9.5 \pm 3.1$ & $9.4 \pm 4.16$ & .53 \\
Mean peak pressure, cm $\mathrm{H}_{2} \mathrm{O}$ & $33.2 \pm 7.65$ & $32.3 \pm 7.65$ & .25 \\
Mean arterial pressure, mm Hg & $84 \pm 13$ & $82 \pm 11$ & .14 \\
Mean heart rate, beats/min & $97 \pm 19$ & $96 \pm 20$ & .81 \\
Mean oxygen saturation, \% & $95 \pm 5$ & $99 \pm 6$ & .29 \\
& & & \\
\hline Data are reported as means \pm SD. & & & \\
FB = flexible bronchoscopy & & & \\
VV = venovenous extracorporeal membrane oxygenation & & & \\
VA = venoarterial extracorporeal membrane oxygenation & & & \\
ECMO = extracorporeal membrane oxygenation & & & \\
& & & \\
\hline
\end{tabular}

pre-FB sweep gas flow was higher in the mixed configuration group $(5.4 \pm 1.9 \mathrm{~L} / \mathrm{min})$ as compared with the VA $(2.8 \pm 1.6 \mathrm{~L} / \mathrm{min})$ and $\mathrm{VV}(4.1 \pm 1.9 \mathrm{~L} / \mathrm{min})(P<.001)$ ECMO groups. Again, no significant differences were observed between the pre- and post-FB sweep gas rates (see Table 3). Ventilator changes before and after FB were also evaluated. Overall, no significant differences in mean $\mathrm{F}_{\mathrm{IO}_{2}}$ $(0.68 \pm 0.23$ vs $0.64 \pm 0.22, P=.09)$, PEEP $\left(9.5 \pm 3.1 \mathrm{~cm} \mathrm{H}_{2} \mathrm{O}\right.$ vs $\left.9.4 \pm 4.16 \mathrm{~cm} \mathrm{H}_{2} \mathrm{O}, P=.53\right)$, and peak airway pressures $\left(33.2 \pm 7.65 \quad \mathrm{~cm} \quad \mathrm{H}_{2} \mathrm{O}\right.$ vs $32.3 \pm 7.65 \mathrm{~cm} \mathrm{H}_{2} \mathrm{O}, P=.25$ ) were noted before and after the FB. Additionally, no significant variations were observed in the pre- and post-FB vital signs, including mean blood pressure, heart rate, and oxygen saturation (see Table 3). These results did not differ when compared by the type of ECMO configuration. FB (BAL and bronchial washings) yielded positive culture data (yeast was excluded) in 37 subjects (47\%), which resulted in a change to the antibiotic regimen in 14 subjects $(38 \%)$ with positive culture data (Table 4).

\section{Complications}

Chest radiographs before and after the procedures were compared: $63.2 \%$ remained unchanged, $22.2 \%$ showed im-
Table 4. Flexible Bronchoscopy Culture Data

\begin{tabular}{lc}
\hline \hline \multicolumn{1}{c}{ Pathogen } & Percentage \\
\hline Pseudomonas & 24 \\
Enterobacter & 12 \\
Klebsiella & 12 \\
Serratia & 10 \\
Methicillin-resistant Staphylococcus aureus & 10 \\
Acinetobacter & 7 \\
Aspergillus & 7 \\
Influenza A & 5 \\
Streptococcus pneumoniae & 2 \\
$\beta$ hemolytic streptococci & 2 \\
Vancomycin-resistant enterococcus & 2 \\
Achromobacter & 2 \\
Methicillin-sensitive S. aureus & 2 \\
\hline
\end{tabular}

provement in opacities, and $14.6 \%$ showed worsening of opacities. In addition, 4 cases of new pneumothorax and 5 cases of worsening of existing pneumothorax were noticed on the radiograph following the procedure. Of the 4 new cases of pneumothorax, one occurred after internal jugular double-lumen cannula placement that preceded the FB, 2 were after FB-guided percutaneous tracheostomy, and one was after thoracentesis before FB. In these cases, the chest radiographs were performed after $\mathrm{FB}$; it is unclear whether pneumothorax was precipitated by FB or due to the preceding procedures. Given that the risk of pneumothorax associated with percutaneous tracheostomy $(0.5-12.5 \%),{ }^{18}$ thoracentesis(4.6-7.8\%), ${ }^{19}$ and internal jugular double-lumen cannula placement $(5-19 \%)^{20}$ is higher than the risk with FB without a trans-bronchial biopsy $(<1 \%),{ }^{21}$ it is possible that the pneumothorax was not a consequence of FB. The presence of preexisting pneumothorax before FB was known in 7 cases. Of these, 5 cases had worsening of existing pneumothorax, and 3 occurred in subjects who underwent BAL. All subjects with known pneumothorax before FB had preexisting small (8.5 French) or medium bore (14 French) chest tube catheters in place before FB. The intraprocedural adverse event rate was low; $96 \%$ of the FBs had no immediate procedural complication. Five subjects had hypoxia during the procedure. Of these, persistent hypoxia (oxygen desaturation to $60 \%$ on pulse oximetry) occurred in one case, requiring abortion of the procedure. Two subjects had moderate hypoxia (70 and $74 \%$, respectively, on pulse oximetry), and another 2 had mild hypoxia ( 82 and $86 \%$, respectively, on pulse oximetry). All of these 4 episodes of hypoxia improved with transient withdrawal of the bronchoscope. Whereas 2 subjects had transient arrhythmias (one bradycardia and one atrial fibrillation), another 2 had reduction in ECMO blood flow due to excessive coughing during the procedure. All of the above events resolved immediately after withdrawal of the bronchoscope. Blood-tinged secretions were noted 
Table 5. Extracorporeal Membrane Oxygenation Outcomes

\begin{tabular}{lcc}
\hline \hline & $\begin{array}{c}\text { ICU Survival in Subjects } \\
\text { Receiving ECMO Who } \\
\text { Underwent FB (\%) }\end{array}$ & $\begin{array}{c}\text { ICU Survival in Subjects } \\
\text { on ECMO Who Did Not } \\
\text { Undergo FB (\%) }\end{array}$ \\
\hline Overall & 75 & 65 \\
VV subjects & 73 & 76 \\
VA subjects & 72 & 60 \\
Mixed configuration & 100 & 67 \\
& & \\
ECMO = extracorporeal membrane oxygenation & \\
FB = flexible bronchoscopy & \\
VV $=$ venovenous extracorporeal membrane oxygenation & \\
VA = venoarterial extracorporeal membrane oxygenation & \\
\end{tabular}

in $21 \%$ of the FBs. Moderate bleeding requiring cold saline instillation occurred in one FB, and minor bleeding requiring no intervention occurred in 4 procedures.

\section{ECMO Outcomes}

The overall ICU survival in our cohort of ECMO subjects who underwent FB was $75 \%$. Subjects supported with mixed ECMO configuration $(100 \%)$ had a higher ICU survival than those with VV (73\%) and VA (72\%) (Table 5). Overall ICU survival for all ECMO subjects at our institution for the study period was $70 \%$.

\section{Discussion}

Fiberoptic bronchoscopy has become an indispensable tool for both diagnostic evaluation and therapeutic intervention in modern day critical care practice. ${ }^{22}$ In most patients, it is a relatively low-risk procedure, and previous studies have demonstrated its safety. ${ }^{15,23}$ However, there are no studies validating the safety of FB in adult patients receiving ECMO. Data from the Extracorporeal Life Support Organization ${ }^{1}$ suggest that the risk of spontaneous pulmonary hemorrhage in subjects receiving ECMO is $8.1 \%$ and may increase to $19 \%$ in subjects undergoing a procedure. Additionally, dislodgement of the ECMO cannula and/or loss of ECMO flow during coughing spells with FB may also occur. ${ }^{24}$

Our study confirms that FB can be safely used in adults supported with ECMO for cardiorespiratory failure. We found that FB in subjects supported with ECMO was not associated with significant worsening of hemodynamic parameters or escalation of ventilator or ECMO support. None of the subjects had a major complication involving the ECMO circuit (ie, cannula dislodgement). Although $21 \%$ of the subjects had bloody secretions after FB, this was self-limited and resolved without any intervention. The majority of chest radiographs remained unchanged after the procedure, although improvement in imaging was observed among subjects who had mucous plugging or copious secretions. As expected, worsening of imaging was observed in subjects who underwent BAL. Previous studies have shown that the prognosis of subjects with respiratory failure due to infections depends on the prompt identification of the causative organisms. ${ }^{25,26}$ In our cohort, FB resulted in positive culture data in $47 \%$ of subjects, and a subsequent change in antibiotic therapy occurred in $38 \%$ of subjects with positive culture data. This is in line with the FB culture yield of $35-71 \%$ reported in studies conducted in critically ill subjects not receiving ECMO. $8,27-30$

Our literature search did not yield any studies evaluating the safety of bronchoscopy in adult ECMO patients. However, our results were comparable with those of 3 studies describing the safety of FB in pediatric ECMO subjects. In 1993, Karlson et al ${ }^{31}$ reported no bleeding complications after FB in 14 pediatric subjects receiving ECMO undergoing FB. In their cohort, $14 \%$ of subjects had worsening of static lung compliance assessed on mechanical ventilation, and $20 \%$ had radiographic worsening after FB. Although we did not notice significant worsening of static lung compliance, $14.6 \%$ of the post-FB chest radiographs worsened. Similarly, in a retrospective study of 79 pediatric subjects receiving ECMO, Kamat et al ${ }^{32}$ showed that FB was associated with low incidence of complications. As in our study, $30 \%$ of the ECMO subjects in their cohort also had blood-tinged secretions, although none had any mild to moderate bleeding episodes. Also, no new pneumothorax was reported in their study. The differences in bleeding between the studies may be related to the populations (adult vs pediatric) studied or the level of anticoagulation used. More recently, Prentice and Mastropietro $^{33}$ demonstrated the safety of FB in pediatric subjects receiving ECMO for cardiac failure. Although their sample size was relatively small, no major bleeding was reported after FB in their cohort. Likewise, FB outcomes and/or complication rates were similar between subjects supported with VA or VV ECMO in our cohort.

Our study is not without limitations. Although our results are based on objective parameters, it is unclear whether any improvement or worsening of radiographic findings after FB is a result of the procedure or rather the underlying disease process. Additionally, given the retrospective nature of the study, results may be biased by documentation or collection errors.

\section{Conclusions}

We conclude that FB can be safely performed in adult patients supported with ECMO. FB is not associated with significant hemodynamics changes, bleeding, and/or mechanical complications during ECMO support. Additionally, FB may improve clinical care by facilitating pulmonary secretion clearance and by possibly increasing 


\section{Flexible Bronchoscopy Is Safe With ECMO}

diagnostic yield and improving antibiotic selection in patients with respiratory infections.

\section{REFERENCES}

1. Paden ML, Conrad SA, Rycus PT, Thiagarajan RR, ELSO Registry. Extracorporeal Life Support Organization Registry Report 2012. ASAIO J 2013;59(3):202-210.

2. Sauer CM, Yuh DD, Bonde P. Extracorporeal membrane oxygenation use has increased by $433 \%$ in adults in the United States from 2006 to 2011. ASAIO journal 2015;61(1):31-36.

3. Cork G, Barrett N, Ntoumenopoulos G. Justification for chest physiotherapy during ultra-protective lung ventilation and extra-corporeal membrane oxygenation: a case study. Physiother Res Int 2014;19(2): 126-128.

4. Guerreiro da Cunha Fragoso E, Goncalves JM. Role of fiberoptic bronchoscopy in intensive care unit: current practice. J Bronchology Interv Pulmonol 2011;18(1):69-83.

5. Vaidya PJ, Leuppi JD, Chhajed PN. The evolution of flexible bronchoscopy: from historical luxury to utter necessity!! Lung India 2015; 32(3):208-210.

6. Montravers P, Gauzit R, Dombret MC, Blanchet F, Desmonts JM. Cardiopulmonary effects of bronchoalveolar lavage in critically ill patients. Chest 1993;104(5):1541-1547.

7. Prebil SE, Andrews J, Cribbs SK, Martin GS, Esper A. Safety of research bronchoscopy in critically ill patients. J Crit Care 2014; 29(6):961-964.

8. Reynolds HY. Bronchoalveolar lavage. Am Rev Respir Dis 1987; 135(1):250-263

9. Trouillet JL, Guiguet M, Gibert C, Fagon JY, Dreyfuss D, Blanchet F, Chastre J. Fiberoptic bronchoscopy in ventilated patients: evaluation of cardiopulmonary risk under midazolam sedation. Chest 1990; 97(4):927-933.

10. Lukomsky GI, Ovchinnikov AA, Bilal A. Complications of bronchoscopy: comparison of rigid bronchoscopy under general anesthesia and flexible fiberoptic bronchoscopy under topical anesthesia. Chest 1981;79(3):316-321.

11. Prakash UB, Offord KP, Stubbs SE. Bronchoscopy in North America: the ACCP survey. Chest 1991;100(6):1668-1675.

12. Pereira W Jr, Kovnat DM, Snider GL. A prospective cooperative study of complications following flexible fiberoptic bronchoscopy. Chest 1978;73(6):813-816.

13. Pallone TL, Silldorff EP, Turner MR. Intrarenal blood flow: microvascular anatomy and the regulation of medullary perfusion. Clin Exp Pharmacol Physiol 1998;25(6):383-392.

14. Muntean W. Coagulation and anticoagulation in extracorporeal membrane oxygenation. Artif Organs 1999;23(11):979-983.

15. Steinberg KP, Mitchell DR, Maunder RJ, Milberg JA, Whitcomb ME, Hudson LD. Safety of bronchoalveolar lavage in patients with adult respiratory distress syndrome. Am Rev Respir Dis 1993;148(3): 556-561.

16. Jolliet P, Chevrolet JC. Bronchoscopy in the intensive care unit. Intensive Care Med 1992;18(3):160-169.
17. Sharma NS, Wille KM, Bellot SC, Diaz-Guzman E. Modern use of extracorporeal life support in pregnancy and postpartum. ASAIO J 2015;61(1):110-114.

18. Fikkers BG, van Veen JA, Kooloos JG, Pickkers P, van den Hoogen FJ, Hillen B, van der Hoeven JG. Emphysema and pneumothorax after percutaneous tracheostomy: case reports and an anatomic study. Chest 2004;125(5):1805-1814.

19. Gordon CE, Feller-Kopman D, Balk EM, Smetana GW. Pneumothorax following thoracentesis: a systematic review and meta-analysis. Arch Intern Med 2010;170(4):332-339.

20. Tsotsolis N, Tsirgogianni K, Kioumis I, Pitsiou G, Baka S, Papaiwannou A, et al. Pneumothorax as a complication of central venous catheter insertion. Ann Transl Med 2015;3(3):40-41.

21. Pue CA, Pacht ER. Complications of fiberoptic bronchoscopy at a university hospital. Chest 1995;107(2):430-432.

22. Raoof S, Mehrishi S, Prakash UB. Role of bronchoscopy in modern medical intensive care unit. Clin Chest Med 2001;22(2):241-261, vii.

23. Hertz MI, Woodward ME, Gross CR, Swart M, Marcy TW, Bitterman PB. Safety of bronchoalveolar lavage in the critically ill, mechanically ventilated patient. Crit Care Med 1991;19(12):1526-1532.

24. Borrelli U, Costa C. ECMO-Extracorporeal Life Support in Adults. Chapter 6, Materials: cannulas, pumps, oxygenators. Sangalli F, Patroniti N, Pesenti A, editors. 2014;72-73.

25. Fagon JY, Chastre J, Domart Y, Trouillet JL, Pierre J, Darne C, Gibert C. Nosocomial pneumonia in patients receiving continuous mechanical ventilation. Prospective analysis of 52 episodes with use of a protected specimen brush and quantitative culture techniques. Am Rev Respir Dis 1989;139(4):877-884.

26. Meduri GU. Ventilator-associated pneumonia in patients with respiratory failure: a diagnostic approach. Chest 1990;97(5):1208-1219.

27. Estella A. [Analysis of 208 flexible bronchoscopies performed in an intensive care unit]. Med Intensiva 2012;36(6):396-401.

28. Jain P, Sandur S, Meli Y, Arroliga AC, Stoller JK, Mehta AC. Role of flexible bronchoscopy in immunocompromised patients with lung infiltrates. Chest 2004;125(2):712-722.

29. Turner JS, Willcox PA, Hayhurst MD, Potgieter PD. Fiberoptic bronchoscopy in the intensive care unit: a prospective study of 147 procedures in 107 patients. Crit Care Med 1994;22(2):259-264.

30. Vélez L, Correa LT, Maya MA, Mejía P, Ortega J, Bedoya V, Ortega H. Diagnostic accuracy of bronchoalveolar lavage samples in immunosuppressed patients with suspected pneumonia: analysis of a protocol. Respir Med 2007;101(10):2160-2167.

31. Karlson KH, Jr., Pickert CB, Schexnayder SM, Heulitt MJ. Flexible fiberoptic bronchoscopy in children on extracorporeal membrane oxygenation. Pediatr Pulmonol 1993;16(4):215-218.

32. Kamat PP, Popler J, Davis J, Leong T, Piland SC, Simon D, et al. Use of flexible bronchoscopy in pediatric patients receiving extracorporeal membrane oxygenation (ECMO) support. Pediatr Pulmonol 2011;46(11):1108-1113.

33. Prentice E, Mastropietro CW. Flexible bronchoscopy for children on extracorporeal membrane oxygenation for cardiac failure. Pediatr Crit Care Med 2011;12(4):422-425. 